\title{
NV centers in silicon carbide: from theoretical predictions to experimental observation
}

H.J. von Bardeleben and J.L. Cantin, Sorbonne Universités, UPMC, Univ. Paris 6, CNRS-UMR 7588, Institut des NanoSciences de Paris, F-75005, Paris, France

Address all correspondence to H.J. von Bardeleben at vonbarde@insp.jussieu.fr

(Received 3 May 2017; accepted 7 July 2017)

\section{Abstract}

NV centers in silicon carbide have been identified in the three main polytypes $3 \mathrm{C}, 4 \mathrm{H}, 6 \mathrm{H}$ by magnetic resonance and photoluminescence experiments and related ab initio calculations. Their properties show them to be promising centers for applications in quantum technology, similar to the case of NV in diamond. However, their spectral range is in the near-infrared, which should allow their integration in telecommunication systems.

The coherent manipulation, measurement, and entanglement of individual solid-state spins using optical fields have recently become a field of high interest in light of their future application for quantum computing. In the past, two different solid-statebased systems have turned out to be promising in this field: optically active quantum dots and solid-state qubits with the NV center, a carbon vacancy nitrogen donor in diamond, being the most advanced one. Numerous achievements have been reported for this center, such as single defect spectroscopy, initialization, and readout of electronic and nuclear spin states at low and room temperatures, entanglement between electron and nuclear spins and electron spin and photons and two-photon quantum interference from distant NV centers. ${ }^{[1-6]}$ These centers possess long electron spin coherence times, individually addressable optical transitions and long-live intrinsic quantum memories based on nuclear spins. Nevertheless diamond is not an optimal semiconductor material for integrating the NV centers in technologically relevant systems.

To overcome this problem a number of theoretical predictions have promoted the search for equivalent defects and in particular NV centers in $\mathrm{SiC} .^{[7-9]}$ The authors postulated a number of necessary properties for a defect in order to be suitable for QT applications: (1) the defect must have a bound state with a multiplet groundstate; (2) the groundstate splitting must be large compares to kT; (3) it must have optical transitions, which are pure intracenter like; (4) it must have luminescence for the read out of the qubit state; (5) it must have an optical pumping cycle, which allows the initialization of the spin groundstate. The main arguments for choosing $\mathrm{SiC}$ were: (i) $\mathrm{SiC}$ is a mature microelectronic material with superior electronic properties and integration facilities, and (ii) NV centers in $\mathrm{SiC}$ should have these required magneto-optical properties just as the NV center in diamond.

The microscopic structure of the NV center in diamond is a $\mathrm{V}_{\mathrm{C}}-\mathrm{N}_{\mathrm{C}}$ nearest-neighbor pair, which in the 1-chargestate is a spin $S=1$ center. The corresponding center in $\mathrm{SiC}$ is a $\mathrm{V}_{\mathrm{Si}}-\mathrm{N}_{\mathrm{C}}$ nearest-neighbor pair [Figs. 1(a)-(c)]. As SiC occurs in different polytypes, different varieties of $\mathrm{V}_{\mathrm{Si}}-\mathrm{N}_{\mathrm{C}}$ centers are possible depending on their respective lattice sites. Whereas only one type of $\mathrm{NV}$ center exists in the structural equivalent to diamond [Fig. 2(a)], i.e. the 3C polytype with zincblende structure, the lower symmetry of the hexagonal polytypes introduces more than one NV center depending on the lattice site of $\mathrm{V}_{\mathrm{Si}}$ [quasicubic or hexagonal and the location of the $\mathrm{N}$ neighbor on the $c$-axis (axial $\mathrm{NV}$ center) or in the

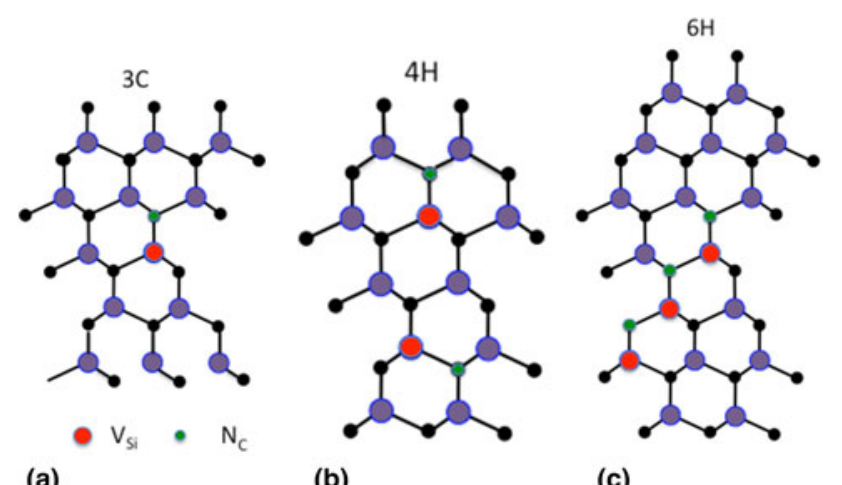

(a)

(b)

(c)

Figure 1. (a) Microscopic structure of the NV center in 3C-SiC (blue and black spheres represent $\mathrm{Si}$ and $\mathrm{C}$ atoms, respectively). (b) Axial and basal NV centers in $4 \mathrm{H}-\mathrm{SiC}$. (c) The three distinct axial NV centers in $6 \mathrm{H}-\mathrm{SiC}$. 


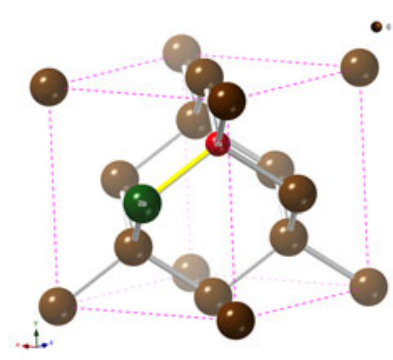

(a)

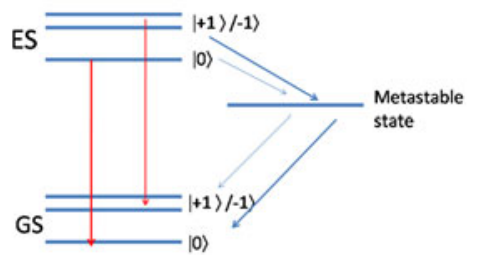

(b)

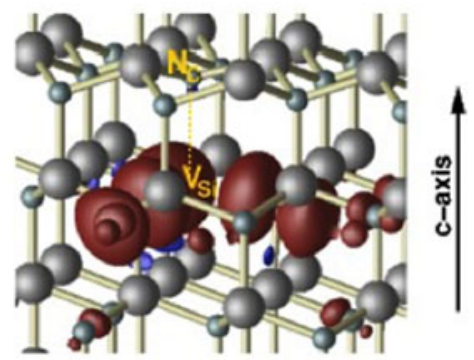

(c)

Figure 2. (a) Microscopic structure of the NV center in diamond. (b) Schematic electronic structure of the NV center in diamond and in 4H-SiC. (c) Spin density distribution of the axial NV(k) center in $4 \mathrm{H}-\mathrm{SiC}$.

$c$-plane (basal NV centers)]. The first ones have $\mathrm{C}_{3 \mathrm{~V}}$ symmetry and are all parallel aligned, whereas the second ones have a lower $\mathrm{C}_{1 \mathrm{~h}}$ symmetry and exist in three magnetically nonequivalent orientations (Fig. 1).

As we have shown recently, all of them have a closely related electronic structure [Fig. 2(b)], which in the 1-chargestate has a spin $S=1$ groundstate and a spin $S=1$ excited state. In the groundstate, the spin density distribution is highly localized on the three carbon nearest-neighbor atoms and has only a weak interaction with the nitrogen atom [Fig. 2(c)]. As the $\mathrm{V}_{\mathrm{Si}}-\mathrm{N}_{\mathrm{C}}$ center in $\mathrm{SiC}$ has different charge transition levels $0 /+, 1-/ 0,2-/ 1-$ and it is the $1-$ charge state, which has the required high-spin properties, Fermilevel engineering is required to assure that all $\mathrm{NV}$ centers are in the 1-charge state. Whereas this is already obtained in the smaller bandgap $3 \mathrm{C}$ polytype in nitrogen-doped n-type samples, an electrical
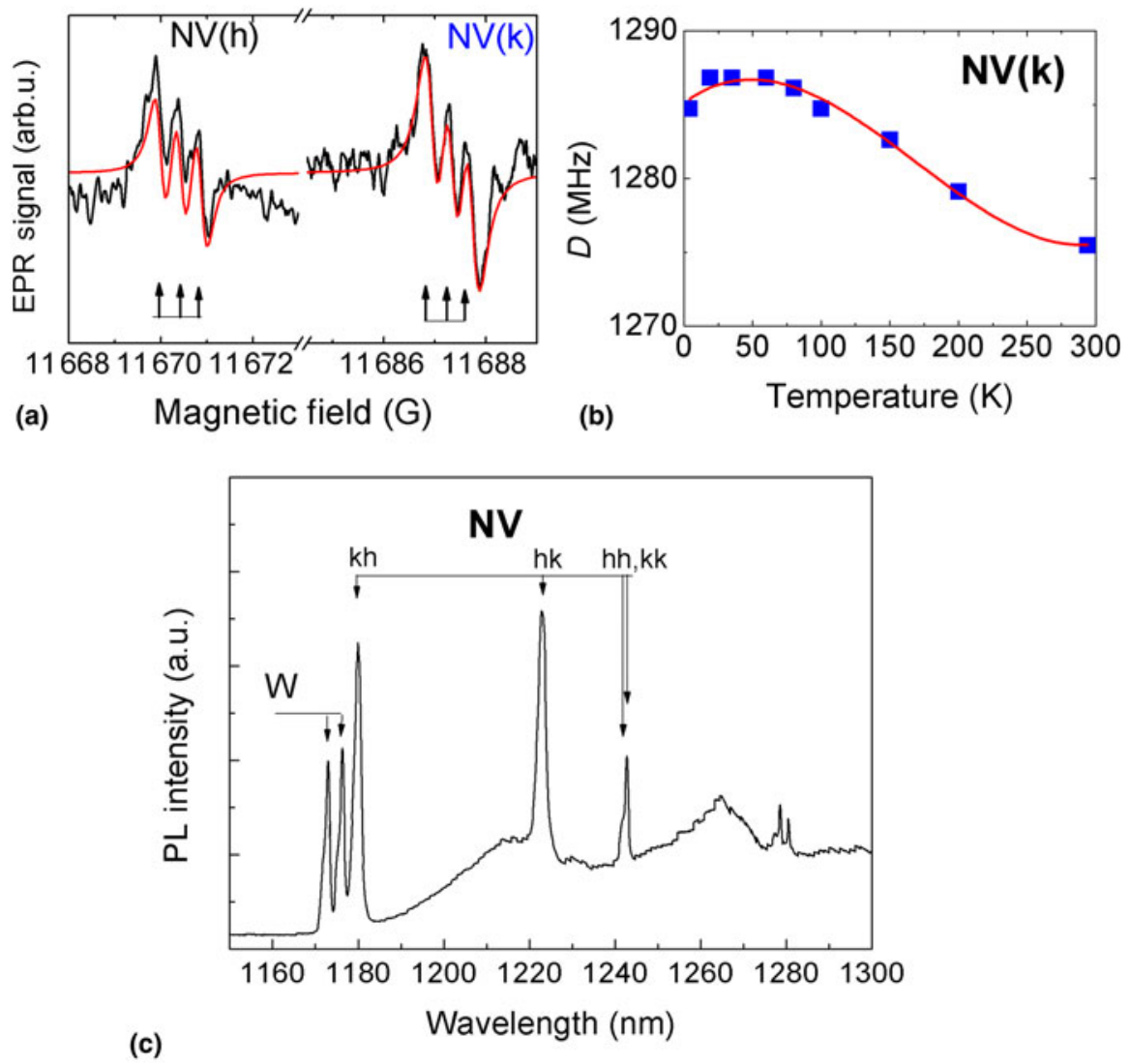

Figure 3. (a) EPR spectrum of the NV(k) center in $4 \mathrm{H}-\mathrm{SiC}$ with resolved ${ }^{14} \mathrm{~N}$ hyperfine structure. (b) Temperature dependence of the zero-field splitting parameter of the NV(k) center in 4H-SiC. (c) ZPL photoluminescence spectra of the four NV centers in 4H-SiC. 
Table I. Spin Hamiltonian parameters of the axial NV centers in SiC. ${ }^{[12]}$

\begin{tabular}{|c|c|c|c|c|c|c|c|c|}
\hline & $g_{L}^{\exp }$ & $g_{\| l}^{\exp }$ & $D^{\exp }$ & $A^{\exp }(1 \mathrm{~N})$ & $A^{\mathrm{DFT}}(1 \mathrm{~N})$ & $A^{\mathrm{DFT}}(6 \times \mathrm{Si})$ & $A^{\mathrm{DFT}}(3 \times \mathrm{Si})$ & $A^{\mathrm{DFT}}(3 \times \mathrm{C})$ \\
\hline $3 C$ & 2.003 & 2.004 & +1303 & 1.26 & -1.08 & 8.0 & 10.3 & 70.3 \\
\hline $4 \mathrm{H} \mathrm{hh}$ & 2.003 & 2.004 & +1313 & 1.23 & -1.09 & 8.14 & 10.36 & 68.97 \\
\hline $4 \mathrm{H} \mathrm{kk}$ & 2.003 & 2.004 & +1270 & 1.12 & -1.15 & 9.05 & 11.05 & 62.70 \\
\hline $6 \mathrm{H} \mathrm{hh}$ & 2.003 & 2.004 & +1328 & 1.32 & -1.08 & 8.41 & 10.32 & 68.52 \\
\hline $6 \mathrm{H} \mathrm{k}_{1} \mathrm{k}_{1}$ & 2.003 & 2.004 & +1278 & 1.21 & -1.20 & 8.26 & 10.42 & 70.44 \\
\hline $6 \mathrm{H} \mathrm{k}_{2} \mathrm{k}_{2}$ & 2.003 & 2.004 & +1355 & 1.26 & -1.06 & 9.01 & 11.11 & 62.18 \\
\hline
\end{tabular}

compensation of n-type $4 \mathrm{H}$ and $6 \mathrm{H}$ samples is necessary to lower the Fermilevel below the shallow donor level.

We have recently shown ${ }^{[10-12]}$ that the expected magnetic and electronic properties of $\mathrm{NV}$ centers in $\mathrm{SiC}$ are in good agreement with the experimental findings. The NV center in $\mathrm{SiC}$ is not a native defect. But as we have demonstrated, the controlled formation of NV centers in the three most important polytypes of $\mathrm{SiC}(3 \mathrm{C}, 4 \mathrm{H}, 6 \mathrm{H})$ can be achieved by particle (electron, proton, and ion) irradiation. The formation of $\mathrm{NV}$ centers is a two-step process: first silicon monovacancy must be generated and in a second step high temperature $\left(800^{\circ} \mathrm{C}\right)$ annealing can be used to form $\mathrm{NV}$ centers by $\mathrm{V}_{\mathrm{Si}}$ diffusion and association with $\mathrm{N}_{\mathrm{C}}$ centers.

Characteristic common features of the NV centers in $\mathrm{SiC}$ are an easily resolved hyperfine interaction of the electron spin with the nuclear spin of the nitrogen neighbor and a narrow photoluminescence zero phonon line (ZPL) [Fig. 3(c)] associated with the spin-dependent recombination between the ${ }^{3} \mathrm{E}-{ }^{3} \mathrm{~A}_{2}$ defect states. ${ }^{[11]}$ A major difference between $\mathrm{NV}$ centers in diamond and $\mathrm{SiC}$ is the spectral range for these optical transitions, which is shifted from the red in diamond to the infrared $(1.2-1.5 \mu \mathrm{m})$ depending on the polytype and lattice sites of $\mathrm{V}_{\mathrm{Si}}$ and $\mathrm{N}_{\mathrm{C}}$ [Fig. 3(c)]. Due to the presence of an intermediate singlet state [Fig. 2(b)] participating in the recombination process, optically induced ground state spin polarization is possible and has been observed in all three cases.

A further important difference in non-isotopically modified $\mathrm{SiC}$ is the presence of two nuclei with non-zero spin: the 4.7 at. $\%{ }^{29} \mathrm{Si}(I=1 / 2)$ and the 1 at. $\%{ }^{13} \mathrm{C}(I=1 / 2)$. Their presence is the reason for increased spin coherence times due to dynamic nuclear decoupling. Further, electronic polarization transfer to either of them is in principle possible.

The ground state spin Hamiltonian parameters of the axial $\mathrm{NV}$ centers in the different polytypes are given in Table I. The experimental values have been mainly obtained from electron paramagnetic resonance (EPR) measurements. They are compared with ab initio calculated values. ${ }^{[12]}$ The good agreement between theory and experimental results has allowed the assignment of the different NV centers in SiC.

In addition to the axial NV centers in which the defects axis is aligned parallel to the $c$-axis, basal NV centers are equally formed by the $\mathrm{V}_{\mathrm{Si}}$ diffusion process. We show in Fig. 4(a) the EPR spectrum of the two types (hk, kh) of basal NV centers in $4 \mathrm{H}-\mathrm{SiC}$. Their point symmetry is lowered to $\mathrm{C}_{1 \mathrm{~h}}$ and they are characterized by a non-zero rhombic zero-field splitting parameter $E$. The calculated zero field splitting (ZFS) parameters are
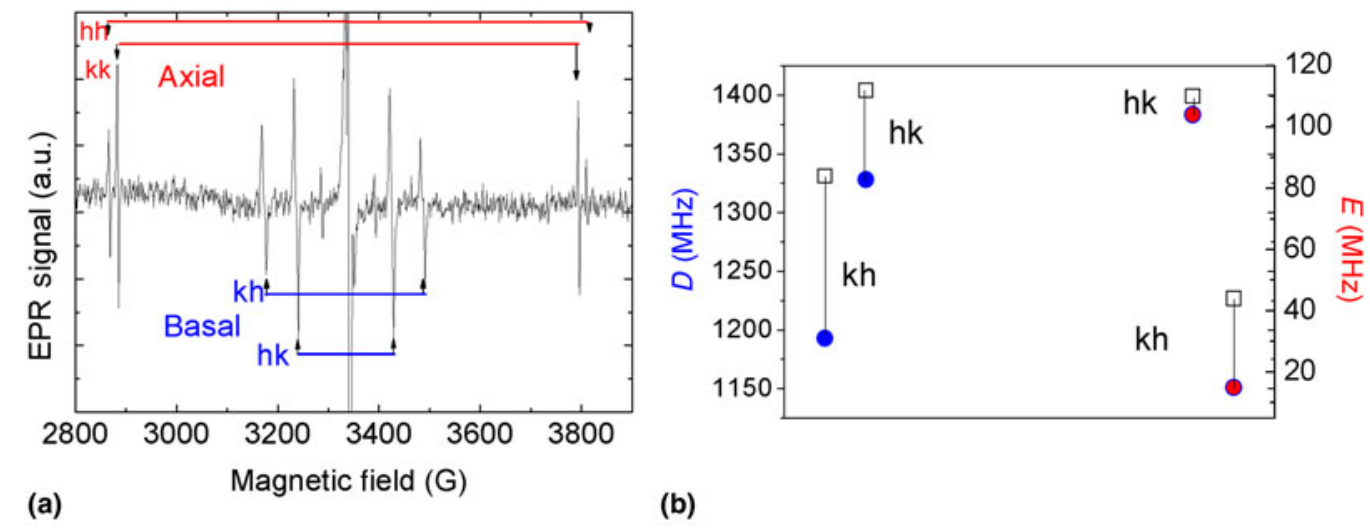

(b)

Figure 4. (a) EPR spectrum of the axial and basal NV centers in $4 \mathrm{H}-\mathrm{SiC}$ for B/c. (b) Experimental (circle) and calculated (square) ZFS parameters $(D, E)$ for the basal NV centers in $4 \mathrm{H}-\mathrm{SiC}$. 

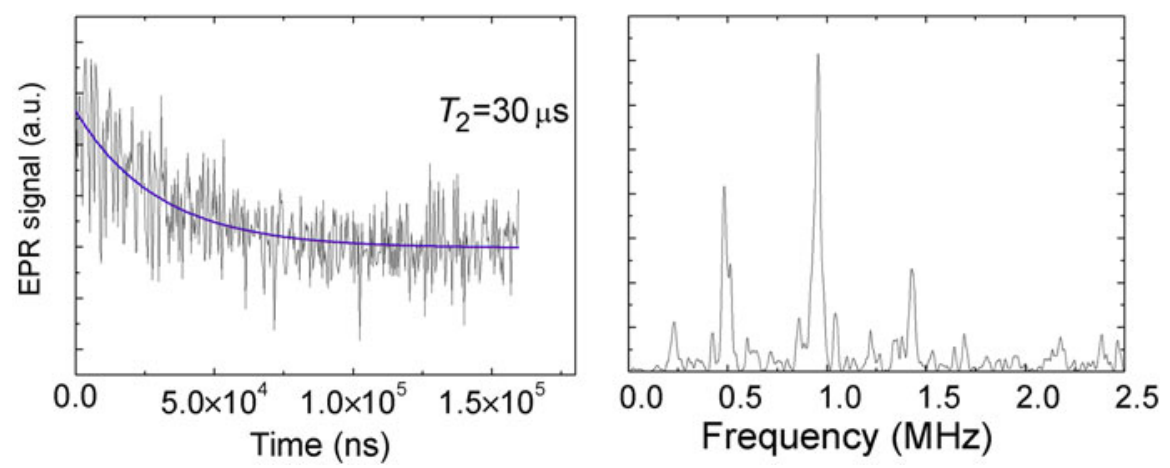

Figure 5. (a) $T_{2}$ measurement of the $\mathrm{ms}=/ 0 \rightarrow 1$ transition of the NV(k) center in $4 \mathrm{H}-\mathrm{SiC} ; T=90 \mathrm{~K}$. (b) ESEEM spectrum displaying the nuclear hyperfine interactions.

in good agreement with the experimental values, but the $D$ values show a systematic shift of $\approx 100 \mathrm{MHz}$ [Fig. 4(b)], which has been attributed to the fact that the spin-orbit coupling contribution has not been considered in these calculations. The basal NV centers have also distinct ZPL photoluminescence lines [Fig. 3(c)] in the near infrared close to the ZPL of the axial centers.

The spin density distribution of the groundstate is strongly localized on the three carbon atoms, which are next to the silicon vacancy and to a less extend on the next-nearest $\mathrm{Si}$ and $\mathrm{C}$ neighbors. It has only a weak localization on the nitrogen atom with a hyperfine coupling constant of typically $1 \mathrm{MHz}$; but due to the small linewidth $(200 \mathrm{mG})$ of the EPR lines in $\mathrm{SiC}$, the hyperfine interaction is nevertheless resolved in the EPR spectra [Fig. 3(a)]. This characteristic feature allows a simple distinction between $\mathrm{NV}$ centers and divacancy centers $\left(\mathrm{V}_{\mathrm{Si}} \mathrm{V}_{\mathrm{C}}\right)^{\circ}$, which have similar zero-field splitting parameters. As in the case of diamond, the zero-field splitting parameter $D$ of the $\mathrm{NV}$ centers in $\mathrm{SiC}$ shows temperature dependence [Fig. 3(b)], which allows their application as a nanoscale temperature sensor.

The spin coherence times of NV centers are expected to be long: first results in $4 \mathrm{H}-\mathrm{SiC}$ show values of $T_{2}=30 \mu$ s at $T=90$ $\mathrm{K}$ [Figs. 5(a) and 5(b)]. Much longer times in highly diluted samples can be expected at low temperature. Single defect spectroscopy and single photon emission has not yet been shown for $\mathrm{NV}$ centers in $4 \mathrm{H}-\mathrm{SiC}$, but have been demonstrated for the closely related $\mathrm{V}_{\mathrm{Si}}-\mathrm{V}_{\mathrm{C}}$ centers ${ }^{[13]}$; similar achievements can be expected for NV centers shortly. Then, entanglement of spin-spin and spin-photons ${ }^{[14]}$ will become accessible with the $\mathrm{NV}$ centers. The superior material properties of $\mathrm{SiC}$, which can be obtained as n-type conductive, $\mathrm{p}$-type or semi-insulating epitaxial layers as well as its demonstrated nanostructuring should allow finally to integrate all functionalities on a single SiC chip.
2. L. Childress, J.M. Taylor, A.S. Sorenson and M.D. Lukin: Fault tolerant quantum repeaters with minimal physical resources and implementations based on single photon emitters. Phys. Rev. A 72, 052330 (2005).

3. L. Childress, M.V.G. Dutt, J.M. Taylor, A.S. Zibrov, F. Jelezko, J. Wrachtrup, P.R. Hemmer and M.D. Lukin: Coherent dynamics of coupled electron and nuclear spin qubits in diamond. Science 314, 281 (2006).

4. J. Wrachtrup and F. Jelezko: Processing quantum information in diamond. J. Phys. Condens. Matter 18, S807 (2006).

5. M.V.G. Dutt, L. Childress, L. Jiang, E. Togan, J. Maze, F. Jelezko, A. S. Zibrov, P.R. Hemmer and M.D. Lukin: Quantum register based on individual electronic and nuclear spin qubits in diamond. Science 316, 1312 (2007).

6. E. Togan, Y. Chu, A.S. Trifonov, L. Jiang, J. Maze, L. Childress, M.V. G. Dutt, A.S. Sorenson, P.R. Hemmer, A.S. Zibrov and M.D. Lukin: Quantum entanglement between an optical photon and a solid-state qubit. Nature 466, 09256 (2010).

7. D. DiVincenzo: Better than excellent. Nat. Mater. 9, 468 (2010).

8. A. Dzurak: Quantum computing: diamond and silicon converge. Nature 479, 47 (2011).

9. A. Boretti: Optical materials: silicon carbide's quantum aspects. Nat. Photonics 8, 88 (2014).

10. H.J. von Bardeleben, J.L. Cantin, E. Rauls and U. Gerstmann: Identification and magneto-optical properties of the NV center in $4 \mathrm{H}-$ SiC. Phys. Rev. B 92, 064104 (2015).

11. S.A. Zargaleh, B. Eblé, S. Hameau, J.L. Cantin, L. Legrand, M. Bernard, F. Margaillan, J.S. Lauret, J.F. Roch, H.J. von Bardeleben, E. Rauls, U. Gerstmann and F. Treussart: Evidence for near infrared photoluminescence of nitrogen vacancy centers in 4H-SiC. Phys. Rev. B 94, 060102(R) (2016).

12. H.J. von Bardeleben, J.L. Cantin, A. Csoré, A. Gali, E. Rauls and U. Gerstmann: NV Centers in $3 \mathrm{C}, 4 \mathrm{H}$, and $6 \mathrm{H}$ Silicon Carbide: a variable platform for solid state qubits and nanosensors. Phys. Rev. B 94, 121202 (2016).

13. D.J. Christle, P.V. Klimov, C.F. de la Casas, K. Szasz, V. Ivady, V. Jokubavicius, J. ul Hassan, M. Syväjärvi, W.F. Koehl, T. Ohshima, N. T. Son, E. Janzén, A. Gali and D.D. Awschalom: Isolated electron spins in silicon carbide with millisecond coherence times. Nat. Mater. 14, 160 (2015).

14. S. Economou and P. Dev: Spin-photon entanglement interfaces in silicon carbide defect centers. Nanotechnology 27, 504001 (2016).

\section{References}

1. M.W. Doherty, N.B. Manson, P. Delaney, F. Jelezko, J. Wrachtrup and L.C. L. Hollenberg: The nitrogen-vacancy color center in diamond. Phys. Rep. 528, 1 (2013). 